\title{
Empirical modelling of site-specific errors in continuous GPS data
}

\author{
Michael Moore • Christopher Watson • Matt King • \\ Simon McClusky • Paul Tregoning
}

Received: 11 November 2013 / Accepted: 13 May 2014 / Published online: 3 June 2014

(C) Springer-Verlag Berlin Heidelberg 2014

\begin{abstract}
Continuous global positioning system (GPS) stations propagate biases and spurious signals into the derived parameter time series when the measurements are subject to site-specific effects, such as multipath. This is a particular problem in the investigation of geophysical and atmospheric phenomena where signals may be small in magnitude. A methodology to remove these erroneous signals from longterm time series will significantly increase the usefulness of the derived time series. This work provides the theoretical basis for use of an empirical site model (ESM) derived from post-fit phase residuals to mitigate unmodelled sitespecific errors. Additionally, we also investigate the effectiveness of applying an ESM to a regional GPS network and a short baseline solution. Under most observing scenarios, we show that the ESM approach is predicted to improve the precision and accuracy of the site coordinates. However, it is important to note that we found some scenarios where the ESM can introduce a bias. For instance, when the antenna is mounted close to the ground. In this scenario, for a short baseline, we observed the introduction of a 4-mm bias in height. Use of an ESM for the same short baseline with an uncalibrated radome substantially improves the results by removing a large bias of over $10 \mathrm{~mm}$ in height. Similarly, application of an ESM derived from historic data yields similar improvements. This demonstrates that the ESM can be a powerful tool when applied to appropriate site-specific con-
\end{abstract}

\section{Moore}

Geoscience Australia, Canberra, ACT, Australia

M. Moore $(\varangle) \cdot \mathrm{S}$. McClusky $\cdot$ P. Tregoning

Research School of Earth Sciences, Australian National University, Canberra, ACT, Australia

e-mail: michael.moore@anu.edu.au

C. Watson · M. King

Surveying and Spatial Science Group, School of Geography and Environmental Studies, University of Tasmania, Hobart, Tasmania, Australia figurations and could potentially be implemented in routine GPS analysis for a broad range of applications.

Keywords Multipath $\cdot$ Site-specific biases $\cdot$ Carrier phase residuals

\section{Introduction}

A large component of GPS site-specific error is caused by unmodelled multipath effects (Elosegui et al. 1995; Hatanaka et al. 2001; King and Watson 2010). Errors due to multipath have a sidereal periodicity (Choi et al. 2004; Agnew and Larson 2007) and can propagate into long-term position time series with significant power at the GPS draconitic year ( 351.4 days), and its harmonics (Ray et al. 2008). Especially when correlated with other parameters of interest, unmodelled error sources can have a detrimental effect of introducing spurious artefacts into parameter time series. Correction of systematic site-specific errors can significantly reduce the phase noise. This yields better resolution of the coordinate time series and more accurate estimates of parameters and interpretation of geophysical signals.

A multitude of techniques have been developed to address the problem of modelling and mitigating multipath effects. Each of these has potential to reduce the effects of multipath on GPS analysis, but they are mostly not widely applicable.

A ray-tracing approach has been developed by Byun et al. (2002) and Lau and Cross (2007) to model and remove the errors from multipath reflections, taking into account complex ray paths and diffraction. Both of these studies use physical models based on the geometric theory of diffraction (GTD), which is an extension of geometrical optics. Byun et al. (2002) point out that use of a simulator based on GTD is only valid for wavelengths which are small in compari- 
son to the dimensions of the interacting objects, and will fail to account for the scattering from small objects. The presence of an object in the near-field of an antenna can induce currents which alter the antenna's characteristics and are not addressed by optical theory (e.g. Elosegui et al. 1995; Byun et al. 2002).

Despite these limitations, the ray-tracing approach could account for a significant portion of multipath effects, if an accurate information is available about the location and microwave characteristics of all nearby objects. Unfortunately, this is rarely the case, and the local area is rarely invariant in time, making it difficult to accurately apply this technique over long-time periods or to large networks.

One promising technique uses the signal to noise ratio (SNR) to map and estimate the multipath environment (Axelrad et al. 1994; Bilich and Larson 2007), to produce a correction map. The technique has been demonstrated to isolate and remove multipath error sources (e.g. Prüllage 2013; Rost and Wanninger 2009, 2010; Benton and Mitchell 2011). However, in the case of this technique, it is not suitable for correcting long-term historic data sets due to inconsistent measures of SNR from different GPS receivers, which in some cases are too coarse to produce a sufficiently accurate correction.

An approach developed by Wübbena et al. (2006) and later refined by Dilßsner et al. (2008) involves calibrating as much of the monumentation with the antenna, during the estimation of the PCV. This may be a promising approach for dealing with the near-field effects, especially if the robotic calibration system utilised can handle heavier payloads. But, this technique does not address site-specific error from farfield effects.

Wübbena and Schmitz (2011) overcome far-field multipath effects by applying an in-situ calibration technique that uses a local network of temporary stations. This requires the placement of temporary stations, each with their antenna and mounts calibrated, around an existing station in a low far-field and near-field multipath environment. This technique is very promising, but like the ray tracing approach, it is not applicable to historic data sets where the equipment or environment of sites has changed.

A number of multipath mitigation techniques attempt to take advantage of the sidereal periodicity of GPS satellite orbits (e.g. Bock et al. 2000) to predict and correct the multipath error. These are enhancements to this technique that factor in variation of the satellite orbit period (e.g. Choi et al. 2004; Larson et al. 2007; Ragheb et al. 2007). Choi (2007) have found a modified sidereal filter to be effective for high frequency observations $(1 \mathrm{~Hz})$ where multipath signals which have a period of 30-300 s. However when observations with a sampling of $30 \mathrm{~s}$ were used, the technique was highly sensitive to an incorrect repeat period, which increased the likelihood of the multipath error becoming out of phase with the filter, resulting in an amplification of the error. The concept behind this technique is somewhat analogous to the one we present, although there are significant differences in the way the correction is obtained and implemented in the reprocessed solution.

Wu and Hsieh (2010) developed a technique based on the statistical analysis of carrier-phase residuals to obtain a correction model, which is correlated with preceding observations. They found that 6 days of data could be used to remove multipath error from current and future data. They applied the technique to a short baseline of $13.5 \mathrm{~m}$ and a longer baseline of $23.1 \mathrm{~km}$, reporting an improvement of $40 \%$ in height determination.

The approach we investigate uses stacks of undifferenced carrier phase residuals to generate a site-specific correction map, referred to throughout this paper as an empirical site model (ESM). This technique was first presented by Hurst Kenneth et al. (1998), who reported an order of magnitude reduction in the sensitivity of the station's final position solution to the elevation cut-off angle, a reduction in the number of phase observations rejected, and a reduction in the post-fit phase residual RMS. Iwabuchi et al. (2004) showed that ESMs derived from phase residuals processed in a double difference network produced a similar results to the undifferenced approach. This confirms that application of the transformation of double-difference phase residuals to undifferenced phase residuals (Alber et al. 2000). Choi (2007) investigated the use of residual stacking, among other site-specific mitigation techniques to improve the analysis of post-seismic analysis after the 2003 TokachiOki earthquake. Choi (2007) used only 3 days of data to obtain an ESM, applying different resolutions in azimuth and elevation of $1^{\circ}, 3^{\circ}$ and $5^{\circ}$. They found that even a $1^{\circ}$ resolution was too coarse to recover multipath errors below $45^{\circ}$ in elevation (for high monuments). In a recent work, Haines et al. (2013) have mentioned an empirical technique to enhance the IGS antenna models used to monitor the vertical sea floor motion at Platform Harvest. The authors speculated that the technique mitigates the effect of multipath; however, they provide limited information about the ESM methodology and its effectiveness. Desai et al. (2013) used the ESM model approach to verify the suitability of two new GNSS stations to be part of a core reference frame network. They also reported a reduction in the variance of position estimates when the ESM was applied to the solution.

An advantage of the ESM approach is that historical data can be used to produce a site model of a particular station, which can be made available in a common format (e.g. a modified ANTEX file) for use by others. Additionally, the ESM approach can also correct for antenna and radome mismodelling errors which may not be possible from other conventional calibration techniques. This makes it an 
attractive technique for application to long-term time-series analysis.

No attention in the literature has been given to the theoretical basis, optimum design and efficacy of the ESM technique. In this paper, we provide a theoretical basis to assess in which conditions an ESM should be used to model sitespecific errors. We also present a series of simulations that shows the conditions under which the ESM approach is most effective, and also those situations where the technique may fail.

\section{Deriving an ESM}

To create an ESM, we started with the IGS absolute antenna calibration model for the antenna installed on any given site (see Fig. 1a). We then compute a block median (at $0.5^{\circ}$ grid spacing) of undifferenced post-fit phase residuals obtained from each individual station (e.g. Fig. 1b). We have used the GAMIT software (Herring et al. 2010) where the clock error for ground sites and satellites is estimated in a post-fit data editing stage (after the ambiguities have been fixed) to obtain undifferenced, ambiguity fixed, post-fit residuals. There are other alternative techniques to transform double-differenced residuals into undifferenced residuals, e.g. (Alber et al. 2000).

We then take the block median of undifferenced phase residuals and add them to a bi-linear interpolation of the antenna PCV (to match the grid spacing), to obtain the ESM model (see Fig. 1c and Eq. 1).

$$
L C_{\mathrm{ESM}}=2.5457 * L 1_{\mathrm{PCV}}-1.5457 * L 2 \mathrm{PCV}+e_{a z, e l}
$$

where $e$ is the bin averaged LC phase residual ( $\mathrm{mm})$. This is then written into a modified ANTEX file for each station, at $0.5^{\circ}$ intervals for both azimuth and elevation.

We have derived a new ESM for each calendar year, or upon a change of antenna at the station. For grid cells which did not contain any phase residuals, no interpolation was performed. This approach is suitable for our application where we are re-processing data; however, interpolation is advisable if the ESM was to be applied for a different time period to its derivation.

\section{Theoretical basis}

An ESM relies on the hypothesis that in a least squares solution with sufficiently high degrees of freedom, the distribution of the undifferenced phase residuals across azimuth and elevation will approximate the systematic site-specific errors caused predominantly by multipath effects.

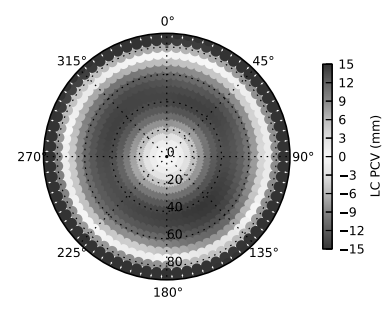

(a) ASH701945C_M LC PCV

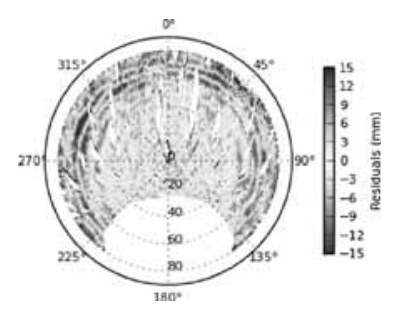

(b) LC block median phase residuals from the station MOBS

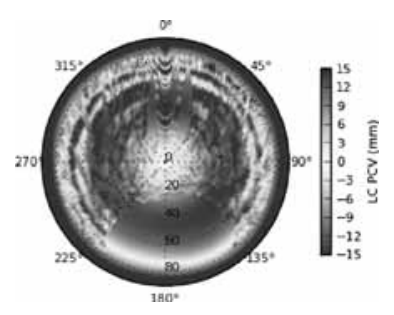

(c) Derived LC ESM for MOBS (Fig 1a + Fig 1b)

Fig. 1 An example of an empirical site model being generated for the IGS station Melbourne (MOBS). The polar plots show azimuth and elevation variation of phase centre variation or phase residuals. a The LC Phase Centre Variation (PCV) for the ASH701945C_M antenna, installed at the station MOBS. b The LC phase residuals obtained from a regional solution of processed data from 2012 for MOBS. c The derived ESM obtained by adding the LC antenna model ASH701945C_M to a block median of the LC phase residuals, which is then stored in a modified ANTEX file

The standard solution by weighted least squares is given by:

$\hat{x}=\left(A^{T} W A\right)^{-1} A^{T} W b$

The design matrix, $A$, is defined by the partial derivatives of the functional model with respect to the parameters being estimated $(\hat{x})$. The matrix $W$ is the inverse of the the observation variance covariance matrix, and $b$ is a vector of observed minus computed.

The residuals $v$ can be calculated from:

$v=A \hat{x}-b$

If we now introduce an unmodelled systematic error $f$ into the observations, the equations now become:

$\hat{x}=\left(A^{T} W A\right)^{-1} A^{T} W(b+f)$

which can be written as:

$\hat{x}=\left(A^{T} W A\right)^{-1} A^{T} W b+\left(A^{T} W A\right)^{-1} A^{T} W f$ 
Thus, $\hat{x}$ is biased by $\left(A^{T} W A\right)^{-1} A^{T} W f$. The least squares residuals with a bias become:

$$
\left.\hat{v}=A\left[\left(A^{T} W A\right)^{-1} A^{T} W b+\left(A^{T} W A\right)^{-1} A^{T} W f\right)\right]-(b+f)
$$

which then leads to:

$$
\hat{v}=A\left(A^{T} W A\right)^{-1} A^{T} W b-b+A\left(A^{T} W A\right)^{-1} A^{T} W f-f
$$

That is, the least squares residuals are not only a function of the original error, $f$, but also its propagation term:

$$
A\left(A^{T} W A\right)^{-1} A^{T} W f-f
$$

Therefore, it could be concluded that re-iterating the solution using an ESM is unlikely to improve the estimated parameters. However, it is important to first understand how the term $A\left(A^{T} W A\right)^{-1} A^{T} W f$ behaves. It is conceivable that, under certain conditions, this term can be small, i.e.

$$
\left\|A\left(A^{T} W A\right)^{-1} A^{T} W f\right\|_{i} \ll\|f\|_{i}
$$

When the propagation term is very small compared to the size of the bias, it follows that the residuals will give a very good approximation of the real site-specific biases and random errors, the latter which average towards zero through combining multiple days of data. The design matrix $A$ and the weight matrix $W$ are the main components controlling the magnitude of the propagation term. The design matrix $A$ will be influenced by the geometry of the stations with respect to the satellite orbits and the parameterisation strategy. The weighting strategy for the observations will influence the matrix $W$.

\section{Simulation}

To investigate the nature of the propagation term of Eq. 8, we use simulations with the specific goal of assessing when carrier-phase residuals are and are not useful to empirically determine site-specific errors. Our GPS simulator is based on the undifferenced GPS observable and is conceptually similar to that of Santerre (1991). We have applied the simulator in a similar way as detailed in King et al. (2003), and further verified in Penna et al. (2007) and King and Watson (2010).

For the tests described here, we have used a typical session span of $24 \mathrm{~h}$, with a sampling period of $120 \mathrm{~s}$. The satellite orbits, clocks and earth orientation parameters have been held fixed. Unless otherwise stated, for the following simulations we estimated coordinates once per 24-h session, tropospheric zenith delays once per hour, the receiver clocks every epoch, and ambiguities were fixed by not estimating them in the solution. We applied elevation-dependent weighting and used a $10^{\circ}$ elevation cut-off angle. We initially adopted an arbitrary station location of $45^{\circ} \mathrm{S}, 140^{\circ} \mathrm{E}$, but explore variations in subsequent analysis. The simulations were run for one $24-\mathrm{h}$ period, with the date set to be January 1, 2012. Random white noise with a magnitude of $1 \mathrm{~mm}$ was added to the $b$ term in Eq. 4 in addition to the systematic error discussed below.

The multipath model added as a site-specific error in the simulations was adapted from the model used by King and Watson (2010). In this paper, we have used derived values for the direct gain $\left(g_{\mathrm{RHCP}}\right)$ for right-hand circular polarised (RHCP) signals and the reflected gain $\left(g_{\mathrm{LHCP}}\right)$ for left-hand circular polarised (LHCP) signal. Signals which are circular polarised change in the direction of polarisation after being reflected, and an antenna's characteristics are significantly different for different types of polarisation. We adopted published values for the Leica AT504 choke ring antenna, commonly deployed in geodetic networks, and of a similar design to the most common geodetic antenna in the IGS network, the AOAD/M_T antenna. We model the multipath effect $\delta \phi_{L}$ by:

$\delta \phi_{L}=\frac{\lambda}{2 \pi}\left(\tan ^{-1} \frac{a \sin \left[4 \pi \frac{H}{\lambda} \sin \varepsilon\right]}{g_{\mathrm{RHCP}}+a \cos \left[4 \pi \frac{H}{\lambda} \sin \varepsilon\right]}\right)$

where $\lambda$ is the carrier phase wavelength for the L1 or L2 carrier-phase signal, and $H$ is the height of the antenna above the reflecting surface. The elevation and zenith angle of the observed satellite is represented by $\varepsilon$ and $z$, respectively. The direct antenna gain pattern $\left(g_{\mathrm{RHCP}}\right)$ was modelled using a simplified modified dipole:

$g_{\mathrm{RHCP}}=\cos \left(z / \mathrm{GR}_{\mathrm{RHCP}}\right)$

We used a direct gain rate value $\left(\mathrm{GR}_{\mathrm{RHCP}}\right)$ of 1.1044 for L1 and 1.0931 for L2 (equivalent to a gain rate of 1.1 for LC). For the reflected gain $\left(g_{\mathrm{LHCP}}\right)$, we used linearly interpolated values for the reflected gain rate $\left(\mathrm{GR}_{\mathrm{LHCP}}\right)$ from a figure of Bedford et al. (2009).

$g_{\mathrm{LHCP}}=\cos \left((\pi / 2) / \mathrm{GR}_{\mathrm{LHCP}}\right)(1-\sin (\varepsilon))$

The amplitude, $a$, of the reflected signal for a given surface roughness $(S)$ was obtained from:

$a=S * \mathrm{GR}_{\mathrm{LHCP}} * R_{a}$

where the values for surface roughness $(S)$ lie between 0 and 1 . A value close to 1 indicates a smooth surface which will preserve the power of the reflected signal, while a value close to 0 indicates a rough surface, where little power is 
returned by the reflected signal. $R_{a}$ is the Fresnel equation for an electric field perpendicular to the plane of incidence and depends on refractive indices $n_{1}$ and $n_{2}$, with $n_{1}=1$ for air, and $n_{2}$ appropriate for the reflecting medium.

$R_{a}=\left[\frac{n_{1} \cos z-\sqrt{n_{2}^{2}-\left(n_{1} \sin z\right)^{2}}}{n_{1} \cos z+\sqrt{n_{2}^{2}-\left(n_{1} \sin z\right)^{2}}}\right]$

Unless otherwise stated we adopted the same values that were used by King and Watson (2010) for $S=0.3$, and $n_{2}=$ 2 , appropriate for a smooth concrete surface surrounding the antenna.

The main limitations of the multipath model described here are the azimuthal symmetry, the time-constant parameters, and the assumption of a homogeneous planar reflector. Next, we test how this simple multipath signal propagates through an undifferenced solution and how effectively it can be recovered again from the least squares residuals.

\subsection{Influence of simulated multipath bias}

The amplitude and frequency of multipath error vary considerably as a function of antenna height above the reflecting surface (Fig. 2). In general, a reflector close to the antenna produces a long-wavelength periodic signal as a function of observed signal elevation angle, whereas a reflector further away will produce a higher frequency multipath bias at low elevation angles and very little bias at high elevation angles.

To assess the ability and efficacy of the ESM approach to recover site-specific errors from least squares post-fit residuals, we calculated the RMS of the difference between the recovered $0.5^{\circ}$ binned ESM and the similarly sampled simulated biases (Fig. 3). We refer to this as the RMS error. Given the random noise in the simulation has amplitude $1 \mathrm{~mm}$, an

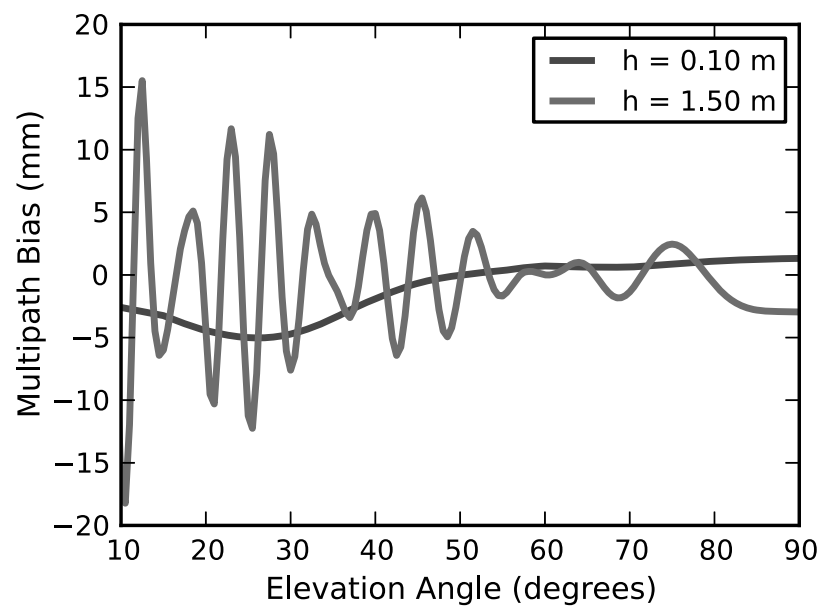

Fig. 2 LC multipath bias as a function of elevation angle, for monument heights of 0.10 and $1.50 \mathrm{~m}$

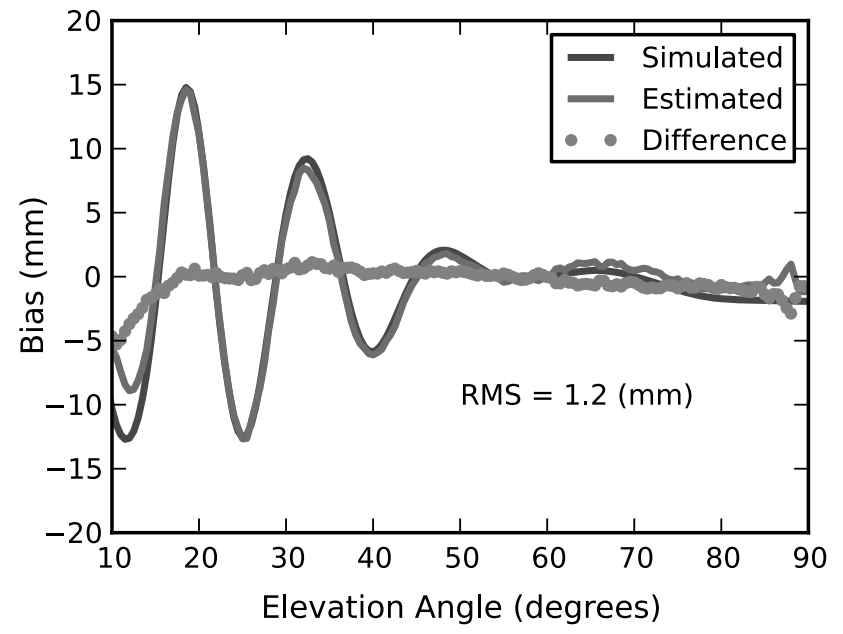

Fig. 3 Simulated LC multipath bias and the ESM multipath bias obtained from the simulated least squares residuals for a monument height of $0.5 \mathrm{~m}$. The difference between the two is plotted in red, from which we calculate the RMS error of the ESM

RMS error of near this value would indicate close to perfect recovery of the simulated multipath (e.g. Fig. 3).

We observe a strong correlation between the RMS error and the monument height (Fig. 4). The RMS error decreases as the monument height increases; however, there is a significant variation for low monuments $(<1 \mathrm{~m})$, and a significant peak in RMS for monument heights close to $0.17 \mathrm{~m}$. From this we infer that the ESM approach has difficulty in recovering multipath signals which vary slowly as a function of elevation angle.

Figure 4 also shows the impact of introducing a higher surface roughness value $(S)$ into the simulated multipath bias. There is a linear increase in the RMS of the difference, between ESM and simulated multipath, for all monument heights as the value of $S$ increases. This indicates that the ESM performance can be significantly degraded, particularly for low monuments close to surfaces which reflect a significant proportion of the incoming signal power.

\subsection{Influence of station location}

We varied the simulated station latitude from $+90^{\circ}$ to $-90^{\circ}$ in increments of $5^{\circ}$. Based on earlier results shown in Fig. 4 , we selected two monument heights. First, we chose $H=1.5 \mathrm{~m}$ as a representation of a high monuments where the ESM is expected to perform well. Second, we chose $H=0.17 \mathrm{~m}$ as a worst-case scenario not uncommon in some networks.

There is no significant latitude dependence from simulated multipath biases for a monument height of $1.5 \mathrm{~m}$; with the opposite the case for $H=0.17 \mathrm{~m}$ (Fig. 5). For monuments mounted close to the ground between the range of $0.17 \pm 0.15 \mathrm{~m}$, the performance of the ESM starts to degrade above $+60^{\circ}$ or below $-60^{\circ}$ in latitude, corresponding with 


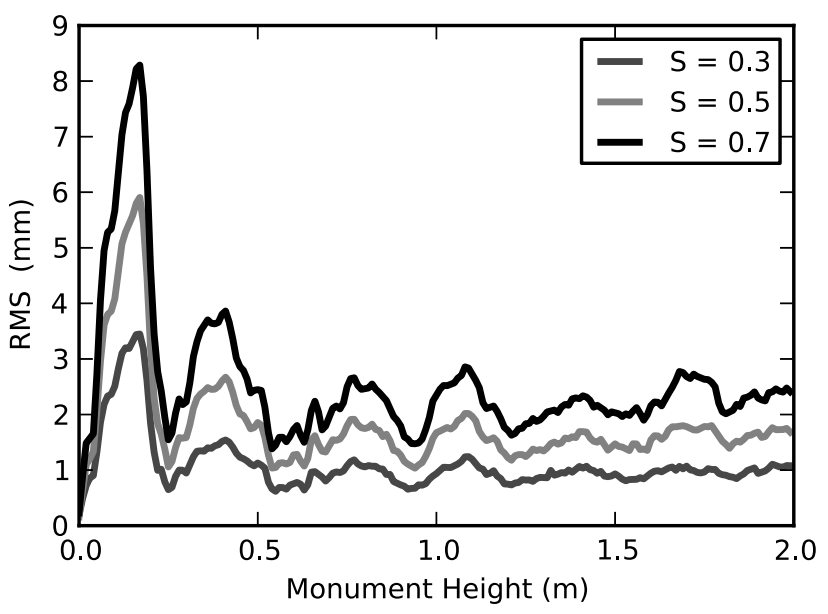

Fig. 4 RMS error of recovering the simulated multipath bias using an ESM for a range of monument heights, and for three different surface roughness $(S)$ values: $0.3,0.5,0.7$. A higher $S$ value indicates that the material will reflect greater input signal power

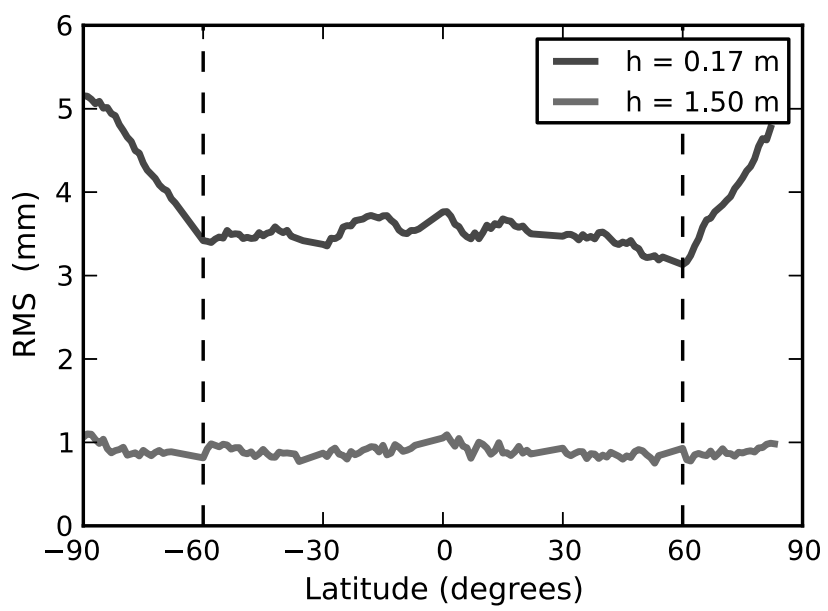

Fig. 5 The influence of a stations latitude and the ability of the ESM to recover the simulated multipath bias for two different monument heights of 0.17 and $1.5 \mathrm{~m}$. The dashed vertical lines are set +60 and -60 in latitude, where we see a poleward increase in RMS for monuments close to a planar reflector

the inclination of the orbit plane. For subsequent simulations we use the default station location of $45^{\circ} \mathrm{S}, 140^{\circ} \mathrm{E}$.

\subsection{Influence of GPS parameterisation}

We investigated the influence of GPS processing parameters on the ESM performance within our simulator. In particular, we looked at the frequency of estimation of receiver clock terms, variation in elevation cut-off angle, changes to the stochastic model used and the frequency of troposphere zenith delay estimation.

Removing the receiver clock estimation term from the simulation is somewhat analogous to simulating a short baseline double-difference baseline processing technique, with multipath only occurring at one of the stations. Despite this simplification it provides a qualitative insight into how the ESM might perform using a different processing technique. We found that there was an overall improvement in performance of the ESM when a baseline approach is used, with the RMS error decreasing by approximately $25 \%$ (Fig. 6a).

Using a PPP scenario, i.e. estimating clocks every epoch, we investigated the impact of the elevation cut-off angle by running the simulations using $5^{\circ}, 10^{\circ}$, and $15^{\circ}$ (Fig. 6b). A $5^{\circ}$ elevation angle significantly increases the magnitude of the peak RMS error for a low monument as well as causing an increase in RMS error for high monuments above $1.8 \mathrm{~m}$. In contrast, the higher elevation cut-off angle of $15^{\circ}$ shows a minor decrease in the peak RMS error, when compared with a $10^{\circ}$ cut-off angle. For higher monument heights there is no significant change in performance, except with a cut-off angle of $5^{\circ}$.

We investigated the effect of different stochastic models of GPS observations upon ESM performance in the simulator by changing the weighting from an elevation-dependent $\left(1 / \sin (e)^{2}\right)$ to a uniform weighting function (Fig. 6c). Applying the latter model produces a small decrease in the peak RMS error, but this comes at a cost of an increase in the RMS error close to $50 \%$ for the rest of the monument heights simulated.

We show the effect of estimation of troposphere parameters upon the performance of the ESM over most monument heights (Fig. 6d). When troposphere estimation is removed there is a significant improvement in the performance of the ESM. The peak RMS error decreased by almost $1 \mathrm{~mm}$, as well as had a shift in the peak RMS to a lower monument height.

\subsection{Impact of multipath on parameter estimates}

We looked at the effect of a simulated multipath bias upon different estimated parameters. Recall that the presented simulations pertain to the geometry on Jan 1, 2012, and do not take into account any time variability of the satellite geometry.

For the standard PPP simulation scenario, where clocks are estimated every epoch and troposphere are estimated every hour, there is a significant height bias of up to $25 \mathrm{~mm}$ for low monument heights $<0.25 \mathrm{~m}$ (blue line of Fig. 7a). Also note that there is a significant height bias for monuments below $1.5 \mathrm{~m}$.

When the same simulation is repeated without troposphere estimation, there is a significant decrease in the peak height error for a small range of low monuments compared to the standard simulation scenario (see red line in Fig. 7a). There remains a significant error for monument heights below 0.1 $\mathrm{m}$ when no troposphere estimates are included. When the simulation scenario is re-run, without clock estimation, but 


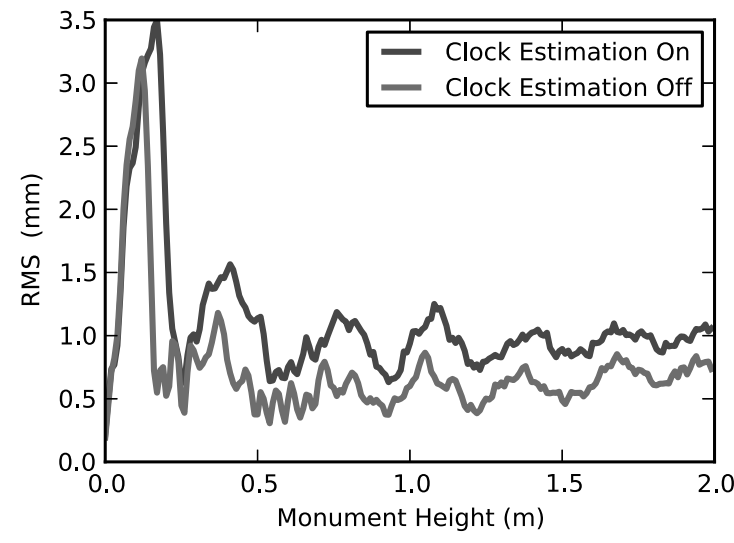

(a) Clock Estimation

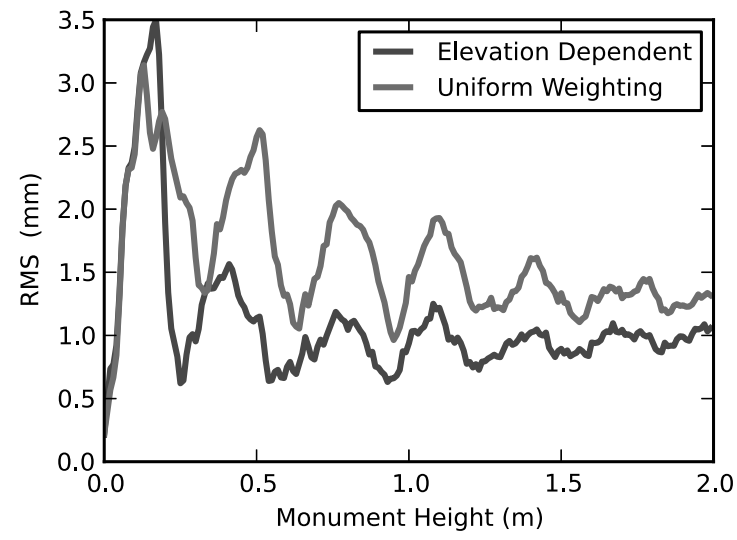

(c) Data Weighting

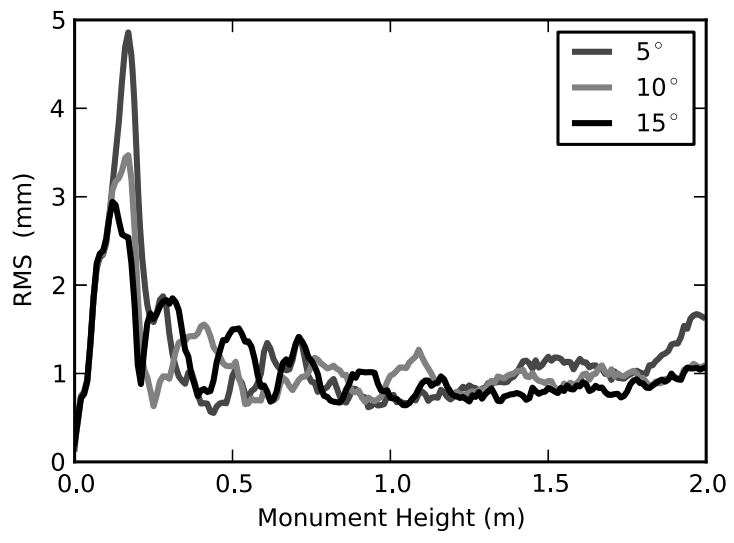

(b) Elevation Cut-off angle

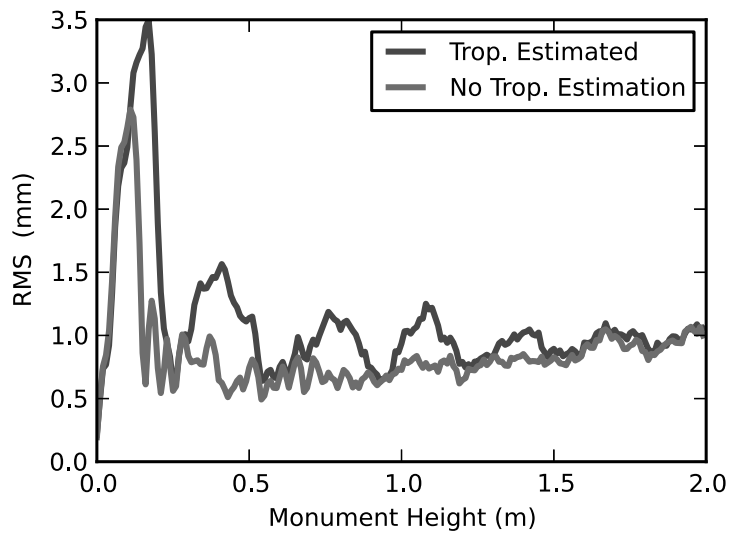

(d) Troposphere Estimation

Fig. 6 Influence of processing variables and estimated parameters on the ESM ability to recover a simulated multipath bias for a range of monument heights

troposphere being estimated every $2 \mathrm{~h}$, we see a further reduction in bias (see black line in Fig. 7a).

Note that the effect of multipath on troposphere estimates will follow the negative correlation between the height and troposphere bias of approximately 3:-1 (Beutler et al. 1987).

To further understand why there is a significant increase in RMS error for low monument heights, we computed the correlation with the estimated height bias, for a range of monument heights, with a simplified elevation-dependant data weighting function and simplified troposphere delay mapping function (Fig. 8).

There is a significant peak in the correlation coefficient for both the troposphere mapping function and elevationdependent weighting function for lower monument heights. This suggests that, for low monument heights, it will be difficult to separate the multipath bias using the ESM approach when a troposphere mapping function or elevation weighting function is applied. Given troposphere mapping functions are integral to geodetic positioning, it therefore appears unlikely from our simulated results that an ESM will yield improved

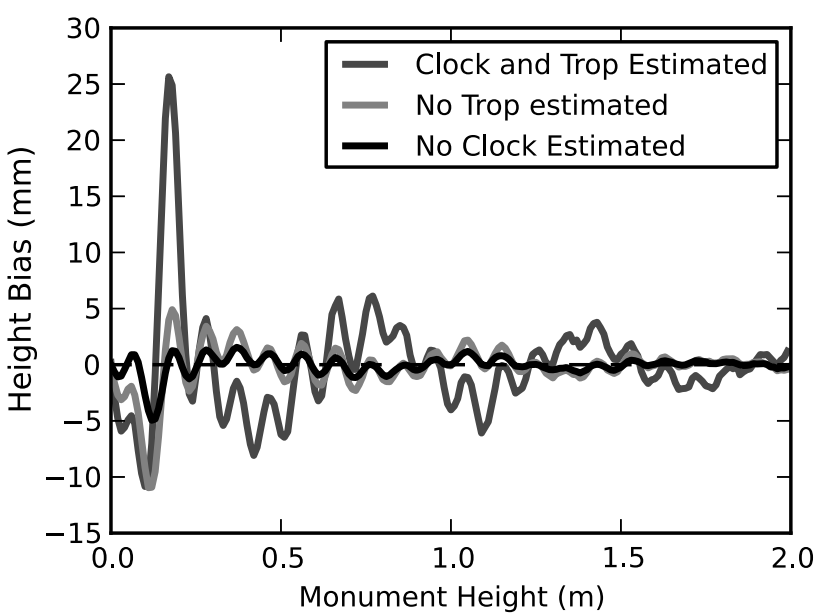

(a) Height Bias

Fig. 7 Effect of multipath bias on the height component for PPP simulation scenario with troposphere and clock estimation (blue line), no troposphere estimation, but with clock estimation (red line), and a simulation with troposphere estimates, but without clock estimation (black line) 


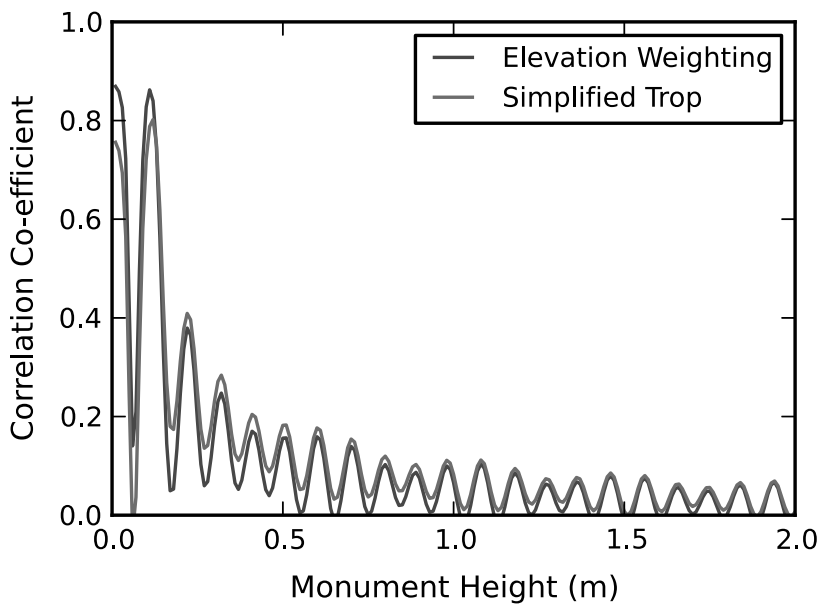

Fig. 8 Correlation coefficient of the simulated height bias, for a range of monument heights, with a simplified troposphere zenith delay mapping function $(1 / \sin (e))$ and a simplified data weighting function $\left(1 / \sin (e)^{2}\right)$

parameter estimates in such cases. We investigated this constraint and a possible means of overcoming it using observed data later in the paper (Sect. 5.3).

\subsection{Impact of applying an ESM on estimation parameters}

Previously, we found that multipath can have a significant effect on height estimates; we now investigate how well the ESM is able to reduce the height bias. First we ran the simulation to create an ESM from the simulated phase residuals, using the methodology outlined in Sect. 2. We then repeated the simulation with the same parameters, except we applied the ESM. We then computed the difference in the estimated absolute height bias from both solutions, where a positive difference indicates an improvement in the solution and a negative value indicates a degradation of the solution (Fig. 9).

The performance of the ESM fell into three distinct categories depending upon monument height. For tall monuments $(>1.2 \mathrm{~m})$, we found that there is little improvement in reducing the height bias through the application of an ESM. For mid-range monuments $(0.25-1.2 \mathrm{~m})$ an average improvement of $1 \mathrm{~mm}$ can be expected and, for a small range of monuments $(0.25-0.18 \mathrm{~m})$, this can reach up to $6 \mathrm{~mm}$ in improvement in the height bias. However, for a small range of low monuments (close to $0.17 \mathrm{~m}$ ) a bias of $2 \mathrm{~mm}$ could be introduced through the application of an ESM.

\subsection{Simulation summary}

Our simulations show that the amplitude and frequency of multipath vary as a function of height above the reflecting surface. Antennae mounted close to a planar reflecting surface have a larger height bias when troposphere mapping

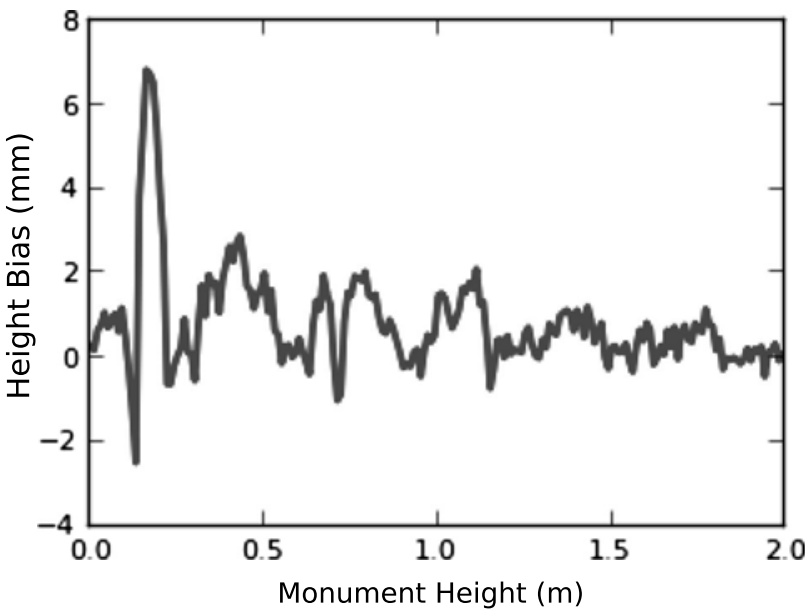

Fig. 9 The difference in height bias, obtained from a simulation with an ESM applied estimated height bias was subtracted from a simulation without an ESM-applied estimated height bias

functions and elevation-dependent weighting is used. This results in the multipath bias being absorbed into troposphere estimates, making it difficult for the ESM to recover the sitespecific bias.

The GPS parameters applied during processing can have an impact on how well the ESM will perform. Estimating clock and troposphere parameters and using a low elevation cut-off angle will decrease the ability of the ESM to recover the multipath bias. Monument heights above $0.25 \mathrm{~m}$ are largely insensitive to the processing parameter set. However, monuments mounted near the ground $(<0.25 \mathrm{~m})$ are sensitive to the processing parameter selected and can have a significant impact upon the error in the derived ESM. We also found that the ESM did not perform as well for stations located above or below the orbit inclination of $\pm 60^{\circ}$ latitude.

The ESM was likely to improve the height bias for most monument heights, particularly between 0.25 and $1.5 \mathrm{~m}$. There was a small subset of monument heights $(\sim 0.17 \mathrm{~m})$ where we found that the ESM actually degrades the solution introducing a further height bias into the time series.

\section{Application to observed GPS data}

Simulations are a convenient tool to assess the performance of an ESM under controlled conditions. To verify our simulation findings, we assessed the performance of applying an ESM to observed data.

\subsection{Regional solution}

We processed observations from a regional network consisting of approximately 60 stations (Fig. 10), unless otherwise stated data processing was carried out as detailed in 


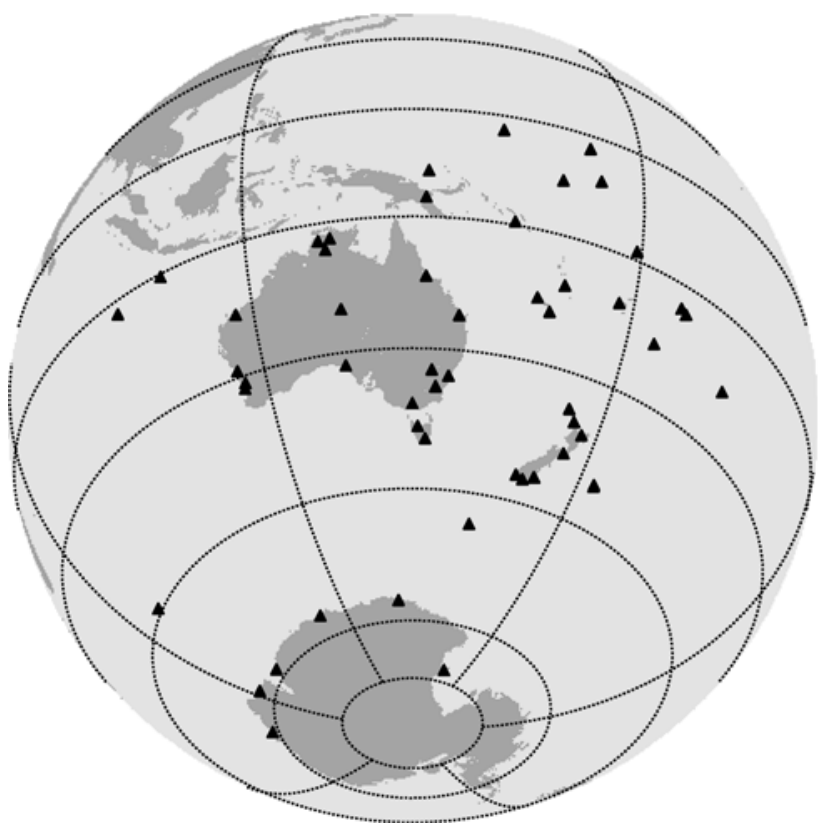

Fig. 10 Map of the regional GPS network processed

Table 1. Data were processed from 2010 through to the end of 2012, where the average ambiguity resolution success rate was $>94 \%$.

\subsubsection{Impact on phase RMS}

We compared the phase RMS obtained from the regional solution with and without the ESM applied. The phase RMS was calculated as a function of azimuth and elevation at $0.5^{\circ}$ bins, any bin which did not have any observations was not included in the calculation. An RMS value was calculated for each station over a period of a calendar year, and whenever there was a change of instrumentation at the station.

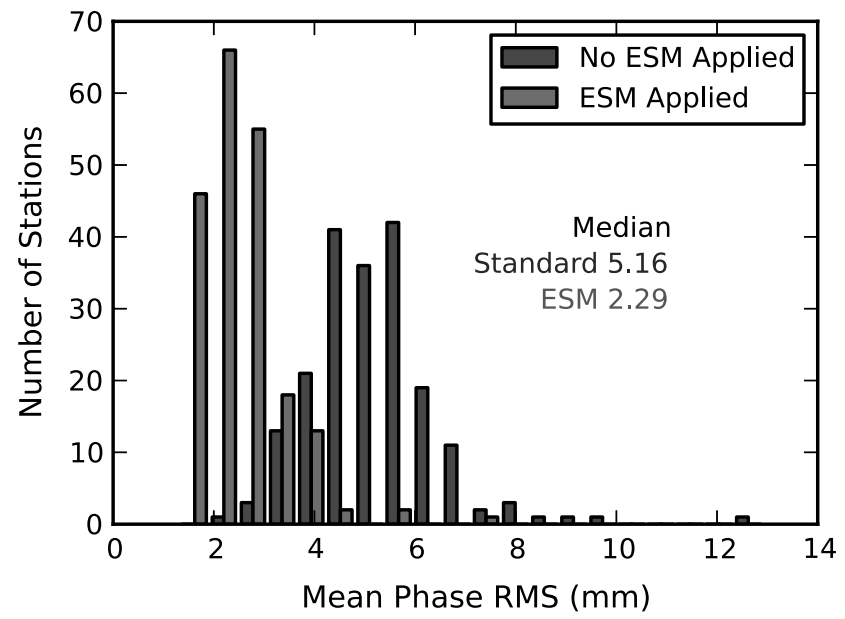

Fig. 11 Histogram of mean phase RMS from regional solutions with and without ESM applied

After applying the ESM, we found a significant reduction in the phase RMS, for all of the stations processed, from a median in the phase RMS of 5.2-2.3 mm (Fig. 11). While this reduction in phase RMS is substantial, we cannot rely on this alone as a measure of the ESM utility. Therefore, we also need to ensure that the estimated parameters are improved in both precision and accuracy.

\subsubsection{Solution spectra}

From the 3 years of processed observations we computed position and velocity estimates for each station, using the same stations to define the reference frame in the solution with and without the ESM. To assess any change in the periodic components of the de-trended coordinates obtained from the regional network solutions we computed the LombScargle periodograms (Scargle 1982). For the majority of sta-

Table 1 Processing parameters applied to short baseline and regional network solutions

\begin{tabular}{lll}
\hline Model/parameter & Track solution & Regional solution \\
\hline Elevation cut-off angle & $10^{\circ}$ & $10^{\circ}$ \\
Software & TRACK (Herring et al. 2010) & GAMIT (Herring et al. 2010) \\
A priori zenith dry delay & MIT & VMF1 (Boehm et al. 2006) \\
A priori zenith wet delay & MIT & VMF1 \\
Antenna phase variations & IGS08 & IGS08 \\
Data rates & 30 (s) & 120 (s) \\
Observation weighting & & Elevation weighting: $a^{2}+\left(b^{2} / \sin ^{2}(e)\right)$ with $a, b$ derived from post-fit phase residuals \\
Orbits & IGS final (fixed) & IGS final (fixed) \\
Solid earth & & IERS2010 \\
Ocean loading & N/A & FES2004 (Lyard et al. 2006) \\
Atmospheric tides & N/A & Tidal and non-tidal applied at observational level (Tregoning and Watson 2009) \\
Ionospheric correction & N/A & second- and third-order IGRF11 (Petrie et al. 2010) \\
\hline
\end{tabular}



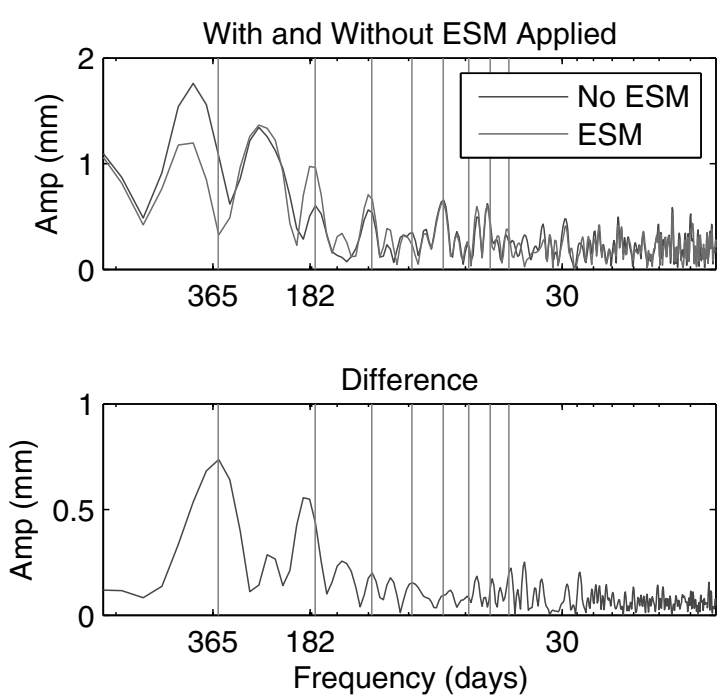

(a) Melbourne (MOBS)

Fig. 12 Station spectra for MOBS (left) and MAW1 (right), for the height component calculated from the de-trended co-ordinate time series produced by the regional solution with and without an ESM

tions analysed there were peaks located at the GPS satellite draconitic year, as has been reported by other authors (e.g. Ray et al. 2008; Collilieux et al. 2007; Agnew and Larson 2007; King and Watson 2010).

We compared the spectra obtained from the regional solution with and without an ESM, as well as calculated the difference in spectra between the two solutions. We did not find any clear underlying pattern; for some stations the solution with the ESM applied had a lower amplitude at the draconitic; and its harmonics (Fig. 12a), while other stations had an increase in amplitudes at the same frequencies (Fig. 12b). This contradicts the findings by Haines et al. (2013), where they reported a decrease in the draconitic harmonics; however, we have used a much larger network of over 60 stations. We did not find a clear relationship between monument height and a change in power at the draconitic, for this data set.

We then stacked the spectra for all of the de-trended coordinate time series obtained from the regional processing with and then without an ESM applied. To obtain a spectral stack we computed the median normalised power estimate across each frequency using the median variance for each frequency bin obtained from the solution without an ESM applied. The median variance calculated from the solution, without the ESM applied, was used to normalise the spectra of the solution with the ESM applied. The ESM solution has a slightly increased power at the draconitic and other harmonics (Fig. 13). The difference of the two solutions also showed a peak at these frequencies. We subdivided stacks of spectra by monument height, but we did not find any clear pattern.
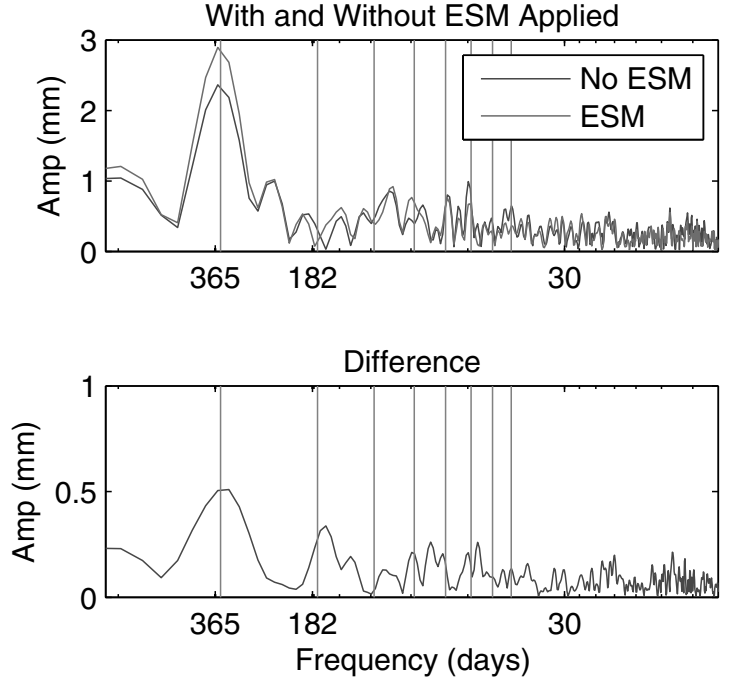

(b) Mawson (MAW1)

applied. The red vertical lines indicate the draconitic period and its harmonics. The spectra of the difference of the two solutions are shown at the bottom

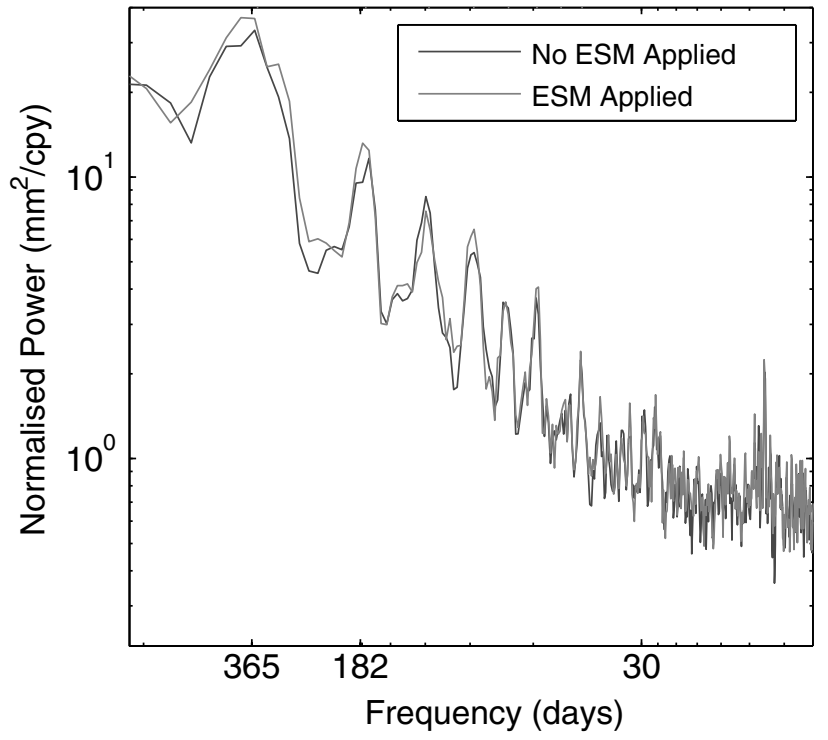

Fig. 13 Spectral stack for the height component of all stations processed in regional network, with and without an ESM applied

We are unable to determine if changes in power at the draconitic frequencies at some stations represent improvements or degradation in the solution with the ESM applied. It is plausible that multipath-induced aliasing at draconitic frequencies interferes constructively or destructively with aliasing at the same frequencies caused by other effects (e.g. errors in sub-daily earth orientation Griffiths and Ray 2013). This combined with the interaction of draconitic-annual and solar-annual terms requires further investigation and outside the scope of this paper. 
From the regional solution we have found that the application of the ESM has reduced the phase RMS. While there are significant changes in spectra at individual stations, we have been unable to identify any pattern for the regional network or any relationship to monument height. We now look at a specific site in detail, where a significant bias is suspected to be present.

\subsection{Local tie anomaly at Yarragadee}

We chose to investigate in detail the performance of applying an ESM at a geodetic facility located at Yarragadee, Western Australia; where there is an IGS station YAR2 and a nearby IGS stations YAR3. There is a local tie residual between YAR2 and the SLR station (7090) of $14 \mathrm{~mm}$ (Altamimi et al. 2011), well outside the Global Geodetic Observing System (GGOS) goals of $1 \mathrm{~mm}$ local ties (Plag and Pearlman 2009). Disagreements in local ties are particularly problematic for the realisation of ITRF (Altamimi et al. 2011).

From our simulations we found that an ESM was unlikely to perform well for antennae mounted close to the ground. Further complicating the situation at YAR2 is the addition of an uncalibrated radome 'JPLA' (Fig. 14). The radome is not concentric with the geometric centre and will add an additional bias to the existing multipath bias. The radome was removed on the 23 of May 2012 (DoY 144) at approximately $03 \mathrm{H} 00 \mathrm{Z}$ and re-installed on 29 of September 2012 (DoY 273) at approximately 00H00Z, as part of an IGS experiment on the impact of uncalibrated radomes (Romero et al. 2011). This provides a rare opportunity to analyse the performance of an ESM over a short baseline, where a controlled equipment change has been made and a high precision survey has been carried out that can be used as ground truth.

We applied the ESM obtained for YAR2 and YAR3 from the 24-h static GAMIT regional solution to a TRACK kine-

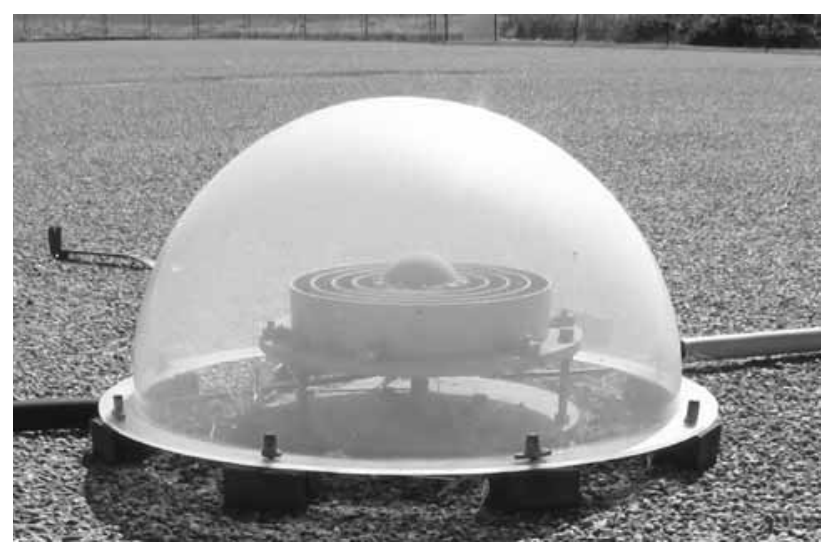

Fig. 14 IGS stations YAR2 with the uncalibrated radome JPLA installed, note that the radome is not concentric with the antenna matic short-baseline solution between YAR2 and YAR3. We switched to a kinematic solution as this will give us an opportunity to apply the ESM derived from a different processing scenario and to be implemented using a different software package; hence allowing us to assess how applicable an ESM may be to other applications. We compared the change in height estimates from the kinematic solution with and without an ESM applied for the different periods when the radome was installed and removed. A new ESM was derived at the start of each calendar year and when the radome was removed or reinstalled. The mean change in height for the different periods and for the different processing scenarios obtained from the kinematic solution and the difference from the terrestrial survey results (Woods and Ruddick 2007) are detailed in Table 2.

The distance between YAR2 and YAR3 is approximately $15 \mathrm{~m}$, and therefore, the effects of geoid slope on such a short baseline will be negligible, which allows us to directly compare the change in height from GPS observations with levelling results.

The kinematic solution with no ESM applied for the radome on period (2012 DoY 001 to DoY 143) has a mean difference of $9.7 \mathrm{~mm}$ from the levelling results. When the radome is removed (DoY 143 to DoY 273) and no ESM applied, the mean difference reduced to $3.1 \mathrm{~mm}$. We infer that the radome is responsible for a 6-mm height bias on the short baseline between YAR2 and YAR3, and the remaining 3-mm bias is due to a combination of far-field and nearfield multipath effects that remain regardless of the radome being installed or removed. These effects may include the mischaracterisation of the antenna phase variation of one or both of the antennas when using a type mean phase centre model.

For the period with the radome on and when the ESM was applied, the height difference from the terrestrial survey is reduced to $0.1 \mathrm{~mm}$. This suggests that the ESM models the bias induced by the JPLA radome, and also takes into account the other site-specific biases. In addition the ESM solution has reduced the height standard deviation by $2 \mathrm{~mm}$

Importantly, however, we observe the introduction of a bias of over $6 \mathrm{~mm}$ in the height component when the ESM is applied during the radome off period. If the true multipath environment is similar to that generated for $H=0.17$ m simulations, then an ESM is derived from historic data at YAR2 and this is not an unexpected result (see Sect. 4.4 and Fig. 7a). We infer that the uncalibrated radome is introducing a larger site bias which is more likely to be correctly modelled by an ESM. However, when the radome is removed the site-specific error changes its characteristics significantly to one which is likely to varying slowly with elevation, and thus poorly removed by the ESM technique. 
Table 2 The mean change in height from YAR3 to YAR2 obtained from kinematic processing of data in 2012, using ESM models derived from 2012 data and 2010 data, and the difference from terrestrial survey results [ $d h=-1.1551 \pm 1 \mathrm{~mm}$ (Woods and Ruddick 2007)]

\begin{tabular}{|c|c|c|c|c|c|}
\hline Radome & & a) No ESM & b) ESM 2012 applied & c) ESM + Constr. TZD & d) ESM 2010 applied \\
\hline \multirow[t]{3}{*}{ On } & Mean (m) & -1.1454 & -1.1550 & N/A & -1.1545 \\
\hline & $\sigma(\mathrm{m})$ & 0.0100 & 0.0080 & N/A & 0.0090 \\
\hline & Diff (m) & 0.0097 & 0.0001 & N/A & 0.0006 \\
\hline \multirow[t]{3}{*}{ Off } & Mean (m) & -1.1520 & -1.1614 & -1.1589 & N/A \\
\hline & $\sigma(\mathrm{m})$ & 0.0100 & 0.0070 & 0.0100 & N/A \\
\hline & Diff (m) & 0.0031 & -0.0063 & -0.0038 & N/A \\
\hline \multirow[t]{3}{*}{ On } & Mean (m) & -1.1456 & -1.1551 & N/A & -1.1568 \\
\hline & $\sigma(\mathrm{m})$ & 0.0100 & 0.0090 & N/A & 0.0090 \\
\hline & Diff (m) & 0.0095 & 0.0000 & N/A & -0.0017 \\
\hline
\end{tabular}

\subsection{Variation to regional processing}

From our simulation results we found that not estimating the troposphere zenith delay estimates decreased the RMS difference between the simulated multipath error, and the error estimated from the least squares residuals by the ESM, and the peak RMS shifted to a lower monument height. The effect was even more dramatic when we investigated the impact upon the height component, with a significant reduction in the peak bias for low monuments.

In a network-based GPS analysis, it is essential to estimate troposphere zenith delay parameters. However, for pairs of nearby stations it is possible to constrain the troposphere zenith delay estimates to one particular station. We investigated whether we could improve the ESM by constraining the troposphere zenith delay estimates at YAR2 to those obtained at YAR3 (once adjusted for the height difference between the two stations), in a new regional solution processed for the radome off period. We then recomputed an ESM from this solution for the station YAR2.

We applied the new ESM to the kinematic solution for the period when the radome was removed, and there was a significant reduction of $2.5 \mathrm{~mm}$ in the bias caused by the ESM (compare column b) and c) Table 2). This agrees with our simulation results where we showed that we can expect a reduction in magnitude of the ESM error, but not necessarily a complete removal of the height bias at the station when troposphere parameters are removed from the solution used to generate the ESM. However, this still does not outperform the solution without an ESM applied, with respect to the mean bias.

\subsection{Application of an ESM derived from historic data}

We have shown that it is possible to apply an ESM derived from a regional network solution and for a particular case successfully apply it to short-baseline kinematic solution.
However, the ESM was derived from the same data set used in the kinematic processing; the ESM technique will be of much more value if historic data could be used to derive an ESM to be applied to current data sets, such as the application within a real-time kinematic network solution.

To test this scenario, we derived an ESM from data processed in 2010 from the same regional network and applied it to the short-baseline kinematic solution for YAR2YAR3 for the radome on period in 2012. Significantly, we found that the level of improvement in the height bias was similar to that achieved using the concurrent data set of 2012 to derive the ESM (Table 2 column b and d). Therefore, ESMs derived from historic data sets may be applicable to future data sets, thus increasing its potential use to a wide range of applications. We anticipate that the ESM derived from the historic data could be further improved through more rigourous interpolation of the phase residuals before being combined with the antenna PCV model.

\section{Conclusion}

We have shown that it is theoretically possible to obtain a model of site-specific biases from residuals of a least squares solution (i.e. an ESM) provided that the bias propagation term is small.

Our simulations demonstrate that the height of the monument, and therefore the characteristics of the multipath bias, has a significant influence on the effectiveness of an ESM to model the bias. For most monument heights, the ESM can recover an accurate model of the bias from the residuals. However for low monument heights, where the multipath bias varies slowly with elevation angle, the ESM can degrade the solution, producing a significant RMS error of up to $4 \mathrm{~mm}$. The ESM is also less likely to be effective for low monuments located at high latitudes $\left( \pm 60^{\circ}\right)$. 
The choice of GPS processing parameters influences how the ESM will perform. The estimation of clock, troposphere parameters and a low elevation cut-off angle will decrease the ability of the ESM to recover the multipath bias.

Application of the ESM to a regional solution significantly reduced the phase RMS of all stations processed from a median of 5.2-2.3 $\mathrm{mm}$. However, this does not demonstrate that the use of an ESM improved estimation of the solution parameters.

An investigation of the spectral characteristics for each station, before and after an ESM was applied, did not reveal any significant pattern. A spectral stack of all stations' power spectral density (PSD) showed a minor increase in normalised power for the draconitic periods, and its harmonics for the time series with an ESM applied. We speculate that any effect at the draconitic frequencies may be masked by constructive or destructive interference with other sources of draconitic aliasing (e.g. Griffiths and Ray 2013)

Application of the ESM to a kinematic solution was effective in the removal of a significant height bias in the baseline YAR2-YAR3. Without application of an ESM, the solution for the radome on period disagreed with the local terrestrial survey by over $10 \mathrm{~mm}$. Whereas, use of the ESM produced a solution that agreed with a local terrestrial survey results to within $1 \mathrm{~mm}$. In suitable site conditions, for instance in a high multipath environment, the site-specific models could potentially be published for use in other applications such as RTK networks. However, it is precautionary to note that during the radome off period the ESM can introduce a bias in the solution (see Table 2).

Additionally, we found that it is possible to use historic data to derive an ESM that could be effectively applied to a different time period. This highlights the potential for ESMs to be produced as part of routine geodetic processing and applied widely to real-time networks.

The ESM technique is an attractive approach that only requires historical data to model site-specific errors. However, the ESM should be applied with care if an antenna is mounted close to a large planar reflector, as in this instance use of the ESM could degrade the solution (although the phase RMS is improved).

Acknowledgments The authors would like to thank Bob King and Thomas Herring for help with modifications to GAMIT and Track for this paper. We acknowledge Paul Cross' comments from past work, which has formed the basis of the theoretical section of this paper and thank the reviewers of this paper, whose comments improved the manuscript. Michael Moore thanks Geoscience Australia for funding his PhD studies at ANU. Data and Products used were obtained from the ARGN and IGS network (Beutler et al. 2009). Processing and analysis of GPS data was carried out on the National Compute Infrastructure (Australia) through time allocation awarded to Geoscience Australia. M.A.K is a recipient of an Australian Research Council Future Fellowship (project number FT110100207).

\section{References}

Agnew D, Larson K (2007) Finding the repeat times of the GPS constellation. GPS Solut 11:71-76

Alber C, Ware R, Rocken C, Braun J (2000) Obtaining single path phase delays from GPS double differences. Geophys Res Lett 27(17):2661-2664. doi:10.1029/2000GL011525

Altamimi Z, Collilieux X, Mtivier L (2011) ITRF2008: an improved solution of the international terrestrial reference frame. J Geod 85:457-473. doi:10.1007/s00190-011-0444-4

Axelrad P, Comp C, MacDoran P (1994) Use of signal-to-noise ratio for multipath error correction in GPS differential phase measurements: methodology and experimental results. In: Proceedings of the 7th international technical meeting of the Satellite Division of The Institute of Navigation (ION GPS 1994), pp 655-666

Bedford L, Brown N, Walford J (2009) Leica AR25 White Paper. Technical report, Leica Geosystems

Benton CJ, Mitchell CN (2011) Isolating the multipath component in GNSS signal-to-noise data and locating reflecting objects. Radio Sci 46(6)

Beutler G, Bauersima I, Botton S, Gurtner W, Rothacher M, Schildknecht T (1987) Accuracy and biases in the geodetic application of the global positioning system. Mitt. Satell.-Beobachtungsstn. Zimmerwald, vol 22, Nr. 22,18 pp

Beutler G, Moore A, Mueller I (2009) The international global navigation satellite systems service (IGS): development and achievements. J Geod 83:297-307. doi:10.1007/s00190-008-0268-z

Bilich A, Larson KM (2007) Mapping the GPS multipath environment using the signal-to-noise ratio (SNR). Radio Sci 42(6)

Bock Y, Nikolaidis RM, de Jonge, PJ, Bevis M (2000) Instantaneous geodetic positioning at medium distances with the global positioning system. J Geophys Res 105(B12):28223-28253. doi:10.029/ 2000JB900268

Boehm J, Niell A, Tregoning P, Schuh H (2006) Global mapping function (GMF): a new empirical mapping function based on numerical weather model data. Geophys Res Lett 33

Byun SH, Hajj GA, Young LE (2002) Development and application of GPS signal multipath simulator. Radio Sci 37(6):10-1-10-23

Choi K (2007) Improvements in GPS precision: $10 \mathrm{~Hz}$ to one day. Ph.D. thesis, University of Colorado, Boulder

Choi K, Bilich A, Larson KM, Axelrad P (2004) Modified sidereal filtering: implications for high-rate GPS positioning. Geophys Res Lett 31

Collilieux X, Altamimi Z, Coulot D, Ray J, Sillard P (2007) Comparison of very long baseline interferometry, GPS, and satellite laser ranging height residuals from ITRF2005 using spectral and correlation methods. J Geophys Res Solid Earth 112(B12):n/a-n/a

Desai S, Gross J, Haines BJ, Stowers DA (2013) Verification and validation of the GNSS stations at the prototype core site for NASAs next generation space geodesy network. In: 2013 Fall AGU Meeting, San Francisco, CA, Poster Presentation G53B-0928

Dilßsner F, Seeber G, Wubbena G, Schmitz M (2008) Impact of nearfield effects on the GNSS position solution. In: Proceedings of the 21 st international technical meeting, ION GNSS-2008, Savannah, pp 612-624

Elosegui P, Davis JL, Jaldehag RTK, Johansson JM, Niell AE, Shapiro II (1995) Geodesy using the global positioning system: the effects of signal scattering on estimates of site position. J Geophys Res 100 . doi:10.1029/95JB00868

Griffiths J, Ray J (2013) Sub-daily alias and draconitic errors in the igs orbits. GPS Solut 17(3):413-422

Haines BJ, Desai SD, Born GH (2013) GPS monitoring of vertical seafloor motion at platform harvest. Adv Space Res 51(8):13691382. (Satellite Altimetry Calibration and Deformation Monitoring using GNSS) 
Hatanaka Y, Sawada M, Horita A, Kusaka M, Johnson J, Rocken C (2001) Calibration of antenna-radome and monument-multipath effect of GEONET-part 1: evaluation of the phase map by GEONET data. Earth Planets Space 53:23-30

Herring TA, King RW, McClusky SC (2010) Documentation for the GAMIT GPS analysis software

Hurst Kenneth J, Bar Server Y (1998) In-situ GPS antenna phase center calibration. In: Proceedings of the Workshop 'GPS/MET Japan Has Come!', pp 54-63

Iwabuchi T, Shoji Y, Sihimada S, Nakamura H (2004) Tsukuba GPS dense net campaign observations: comparison of the stacking maps of post-fit phase residuals estimated from three software packages. J Meteorol Soc Jpn 82(1B):315-330

King M, Coleman R, Nguyen LN (2003) Spurious periodic horizontal signals in sub-daily GPS position estimates. J Geod 77:15-21

King MA, Watson CS (2010) Long GPS coordinate time series: multipath and geometry effects. J Geophys Res 115. doi:10.1029/ 2009JB006543

Larson KM, Bilich A, Axelrad P (2007) Improving the precision of high-rate GPS. J Geophys Res 112

Lau L, Cross P (2007) Development and testing of a new raytracing approach to GNSS carrier-phase multipath modelling. J Geod 81:713-732. doi:10.1007/s00190-007-0139-z

Lyard F, Lefevre F, Letellier T, Francis O (2006) Modelling the global ocean tides: modern insights from FES2004. Ocean Dyn 56:394-415

Penna NT, King MA, Stewart MP (2007) GPS height time series: shortperiod origins of spurious long-period signals. J Geophys Res Solid Earth 112(B2)

Petrie EJ, King MA, Moore P, Lavalle DA (2010) Higher-order ionospheric effects on the GPS reference frame and velocities. J Geophys Res 115

Plag H-P, Pearlman MR (2009) Global geodetic observing system: meeting the requirements of a global society on a changing planet in 2020. Springer, Berlin

Prüllage A (2013) For the calibration and compensation of multipath effects on GNSS stations in the networking SAPOS Lower Saxony. Master's thesis, Jade Hochschule Wilhelmshaven Oldenburg Elsfleth, Department of Applied Geodesy, Oldenburg
Ragheb A, Clarke P, Edwards S (2007) GPS sidereal filtering: coordinate- and carrier-phase-level strategies. J Geod 81:325-335

Ray J, Altamimi Z, Collilieux X, Dam T (2008) Anomalous harmonics in the spectra of GPS position estimates. GPS Solut 12:55-64

Romero N, Rebischung P, Ray J, Schmid R, Fisher S, Griffiths J (2011) The IGS campaign to measure position corrections for uncalibrated IGS radome stations. In: IGS, Workshop 2011, Poland

Rost C, Wanninger L (2009) Carrier phase multipath mitigation based on GNSS signal quality measurements. J Appl Geod 3(2):81-87

Rost C, Wanninger L (2010) Carrier phase multipath corrections based on GNSS signal quality measurements to improve CORS observations. In 2010 IEEE/ION: Position Location and Navigation Symposium (PLANS), pp 1162-1167. IEEE

Santerre R (1991) Impact of gps satellite sky distribution. Manuscripta Geodaetica 16(1):28-53

Scarge JD (1982) Studies in astronomical time series analysis. iistatistical aspects of spectral analysis of unevenly spaced data. APJ 263:835-853

Tregoning P, Watson C (2009) Atmospheric effects and spurious signals in GPS analyses. J Geophys Res 114

Woods A, Ruddick R (2007) The 2007 Yarragadee (Moblas) local tie survey. Technical report, Geoscience Australia

Wu J, Hsieh C-H (2010) Statistical modeling for the mitigation of gps multipath delays from day-to-day range measurements. J Geod $84: 223-232$

Wübbena G, Schmitz S (2011) On GNSS station calibration of nearfield multipath in RTK-networks. In: International Symposium on GNSS, space-based and ground-based augmentation systems and applications, Berlin

Wübbena G, Schmitz M, Boettcher G (2006) Near-field effects on gnss sites: analysis using absolute robot calibrations and procedures to determine corrections. In: Proceedings IGS Workshop, pp 8-12 